\title{
Looking Back with Neutrinos ${ }^{a}$
}

\author{
L. Stodolsky \\ Max-Planck-Institut für Physik, 80805 Munich
}

\begin{abstract}
We briefly discuss the history of suggestions for time- of-flight effects due to nonzero neutrino mass and a recent proposal that such effects can be used to determine the parameters of cosmology. With neutrinos there is potentially a much deeper "look back time" than with photons. We note a new point, that if future large scale neutrino detection arrays see long-time secular variations in counting rate, this could be due to highly redshifted bursts originating in the early universe.
\end{abstract}

\section{Introduction}

Neutrinos are everywhere and they're getting into everything. Some have been in the newspapers and a number of them certainly penetrated the Pentagon and have gone through secrets at Los Alamos. Who knows what they won't get into next?

In view of this interesting if perhaps a bit alarming situation, it shouldn't come as a surprise that they're also getting into history. We all know of course that they play a major role in the history of the universe. Here, however we want to talk about yet another role for them: not as players, but as reporters.

Back at the time of supernova 1987a and even long before, it was realized that if neutrinos had a small mass and if they traveled over astronomical distances the small deviation of their velocity from the speed of light could lead to possibly observable effects. It seems to have begun with Zatsepin 1 , who in 1968 suggested if a neutrino burst from a supernova would be detected it could be used to limit the neutrino mass. He used the fact that a mass would mean that the velocity would be energy dependent. Thus a burst starting out a few seconds wide in time would, due to the range of energies contained in it, spread as it traveled to us to us, and this spreading would reflect on the mass. He concluded one could improve the then-existing laboratory mass limit of 200 $\mathrm{eV}$ to about $2 \mathrm{eV}$. Later, with the discovery of different neutrino types came the realization 2 of an even more obvious effect: given different neutrino types, each one should have a different mass and so a different velocity in a burst. In SN 1987a this lead to a limit 3 of around $10 \mathrm{eV}$ for $\nu_{e}$, unfortunately not very strong by present standards.

We see that a neutrino mass has two potentially observable consequences:

- A burst spreads in time due to the dispersion of velocities.

${ }^{a}$ Presented at the Carolina Symposium on Neutrino Physics, Columbia, March 2000. 
- Different mass states arrive at different times.

Note that one of the mass states could also correspond to photons emitted in the event, in which case $m=0$ and the first point doesn't apply; or contrariwise one of the mass states could be an as yet undiscovered heavy particle (e.g. a WIMP) in which case the time-of-flight effects are magnified. Also note we should expect that the neutrino mass states are generally mixed flavor states, leading to a "flavor-echo", as we discussed in ref [2].

Then in another vein, there is the possibility of some fundamental tests of relativity 4 . The limiting velocity for all forms of radiation should be $c$, the speed of light. There is no reason to doubt this and many good reasons to believe it. Nevertheless, SN 1987a gave us a rare chance to test it in a novel way, and a rather strong confirmation it is, on the $10^{-9}$ level.

\section{Measuring the Universe with Neutrinos}

Here I would like to report on a continuation of this story, which is again nothing but kinematics, but nevertheless with amusing consequences. Once again we are concerned with relativity and neutrino mass. But now it is general relativity and instead of learning about the properties of the neutrino we want to use them to determine the large scale geometry of the universe.

The main point is the following: Neutrinos with mass, when emitted from a distant source will travel somewhat slower than the speed of light. This deviation, however, will depend on the cosmological epoch, since as the universe expands the neutrinos slow down. When the neutrino finally reaches us, its total travel time represents in effect a record of the cosmological epochs it has passed through.

This is a simple idea and could easily have been calculated by the founders of general relativity and cosmology, if they had known about the existence of a very light particle which travels almost, but not quite at the speed of light. But they didn't of course, and so they concentrated on "geometry" such as the measurement of angles subtended by "standard measuring rods" or the apparent brightness of "standard candles". Thus it is only very recently, stimulated by the growing evidence for neutrino mass 0 , that we realized 6 there is a "particle" as opposed to a "geometrical" way of surveying the universe.

Assuming that we know or will know the neutrino masses, it turns that neutrino bursts from sources with identified red shift can give us both the Hubble constant and the acceleration parameter of cosmology $q$. No independent knowledge of the distance to the source is necessary, so difficulties involving the "cosmic distance ladder" are absent.

To see how this comes about, let us calculate the difference in arrival 
times for two mass states emitted in the same cosmic event. We take the standard FRW metric $d s^{2}=d t^{2}-a^{2}(t)(d \mathbf{x})^{2}$, where we define $a(t)$ to be the expansion factor of the universe normalized to its present value: $a(t)=$ $R(t) / R($ now $)$, so that $a($ now $)=1$. We proceed by finding an equation for the coordinate velocity $d x^{i} / d t$, where $x^{i}$ is along the particle's flight direction. First we express $d x^{i} / d t$ in terms of $P^{i}(t)$, the spatial part of the contravariant fourmomentum $m d x^{\mu} / d s$. From the definition of the metric we have $a(t) d x^{i} / d t=$ $\left[a(t) P^{i}(t)\right] / \sqrt{m^{2}+\left[a(t) P^{i}(t)\right]^{2}}$.

Expanding for the relativistic case $P \gg m$ we obtain $a(t) d x^{i} / d t \approx 1-$ $\frac{1}{2} m^{2} /\left[a(t) P^{i}(t)\right]^{2}$ To find $P^{i}(t)$, we now make use of the fact that the covariant or "canonical momentum" $P_{i}$ is constant (since nothing depends on the xcoordinate and $\left.P_{i} \sim \partial_{i}\right)$. Furthermore, since the different kinds of momenta are related through the metric tensor, they all become equal at $t($ now), where $a=1$. Hence we can identify the constant covariant momentum as $P($ now $)$, the momentum at the detector. Thus from $P^{i}=g^{i j} P_{j}=1 /\left(a^{2}\right) P_{i}$, we obtain $P^{i}=1 /\left(a^{2}\right) P($ now $)$. Thus we finally have

$$
\frac{d x}{d t} \approx \frac{1}{a(t)}-a(t) \frac{1}{2} \frac{m^{2}}{P^{2}(\text { now })} .
$$

The first term by itself will be recognized as just the equation for motion along the light cone, and then there is a small correction involving the mass.

Introducing $\Delta x$ for the difference in the $x$ coordinate of two different particles of mass $m_{2}$ and $m_{1}$ emitted in the same event at the same time

$$
\frac{d(\Delta x)}{d t} \approx a(t) \frac{1}{2}\left[\frac{m_{1}^{2}}{P_{1}^{2}(\text { now })}-\frac{m_{2}^{2}}{P_{2}^{2}(\text { now })}\right]
$$

At the present epoch with $a=1, \Delta x$ is just the spatial separation of the two particles. Integrating, we have for this separation, or in view of $v \approx c=1$ for the time difference in arrival at a detector

$$
\Delta t \approx \Delta x \approx \int a(t) d t \frac{1}{2}\left[\frac{m_{1}^{2}}{P_{1}^{2}(\text { now })}-\frac{m_{2}^{2}}{P_{2}^{2}(\text { now })}\right] .
$$

It is thus $\int a(t) d t$ which "records" the cosmological information. Observe $a$ is small at early times so that most of the effect comes near the present time, as expected since this is when the neutrinos are the "slowest". The expressions in the brackets are the familiar factors giving the difference in velocity for highly relativistic particles.

It now only remains to get rid of the coordinate dependent $a$ and $t$ and to re-express things in terms of an observable, namely the red shift parameter $z$ 
for the event. With the expansion of $a(t)$ for recent epochs $a(t)=1+H[t-$ $t($ now $)]-\frac{1}{2} q H^{2}[t-t(\text { now })]^{2}+\ldots$, and the redshift parameter $z=1 / a-1=$ $-H[t-t($ now $)]+(1+q / 2) H^{2}[t-t(\text { now })]^{2}+\ldots$, we find

$$
\Delta t \approx \frac{z}{H}\left[1-\frac{3+q}{2} z+\ldots\right] \frac{1}{2}\left[\frac{m_{1}^{2}}{P_{1}^{2}(\text { now })}-\frac{m_{2}^{2}}{P_{2}^{2}(\text { now })}\right]
$$

giving the result in terms of the directly observable $z$. We thus have the measured quantities for an event, $\Delta t$ and $z$, given in terms of the present Hubble constant $H$ and the acceleration parameter $q$. Thus in principle two good events-assuming the neutrino masses well known by the time this all happens- fix these cosmological parameters.

This was for the time delay between two distinct mass states. However it may well be that the mass differences aren't big enough to give cleanly separated pulses. In that case we can try using the pulse spreading effect. So consider the same mass but different momenta $P$ and $P^{\prime}$. We then get a time delay between the two of

$$
\Delta t \approx \frac{z}{H}\left[1-\frac{3+q}{2} z+\ldots\right] \frac{1}{2} m^{2}\left[\left(\frac{1}{P(\text { now })}\right)^{2}-\left(\frac{1}{P^{\prime}(\text { now })}\right)^{2}\right] .
$$

These formulas are low $z$ expansions, to order $z^{2}$.

The first term in $z$ in the expressions is just what we would get without general relativity; it says that $\Delta t$ is simply the velocity difference times the distance (since $z=H d$ is just the Hubble law, where $d$ is the distance). Even this is not entirely trivial, however, since it shows how with neutrinos one can find $\mathrm{H}$ without knowing the distance to the object. That traditional difficulty of observational cosmology, the "cosmological distance ladder", is gone. It is of course replaced by the difficulty of detecting neutrino bursts at cosmological distances. If the neutrino observations are ever made it will certainly be interesting to compare the "neutrino $H$ " found this way with the "photon H" found by astronomy.

\section{$3 \quad$ Reality?}

The realization of these proposals, attractive as they might be, does not seem immediate or certain. First of all, we need a class of cosmic events that emits bursts of neutrinos, or neutrinos and photons simultaneously and with great intensity. This does not seem impossible, and certainly hadronic mechanisms for phenomena like the gamma ray bursts would do this, giving neutrinos from charged pions and photons from neutral pions. 
Then there is the question of detectability. With the bursts coming from cosmological distances, they will reach us substantially weakened. Here it seems we must rely on the development of the $\mathrm{km}$ scale detectors in the ocean and in the ice.

Assuming all this is accomplished we have the question of the good separation of the pulses. If our effects are too small compared to the duration of the original event itself, our time of flight effects will be lost or at least require an elaborate statistical analysis to be extracted.

To get some feeling for this we can evaluate the kinematic factor in front of the formulas in terms of an $\mathrm{eV}$ (mass) ${ }^{2}$ for the neutrinos and $\mathrm{GeV}$ for their energy:

$$
\frac{(m / \mathrm{eV})^{2}}{2(P / \mathrm{GeV})^{2}} \approx 50 \mu \mathrm{sec} / \mathrm{Mpc}
$$

It appears that even at a thousand Mpc, a substantial part of the way across the visible universe, we may only expect some msec delays. While msec or even $\mu$ sec speed doesn't seem very difficult for particle detectors, there is the problem of the intrinsic time scale of the burst itself. If we take supernovas or gamma-ray bursts as a guide, where the timescale is on the order of some seconds, then it seems that to have distinctly separated bursts we would need to have particles distinctly more massive than eV's. One possibility might be the third neutrino mass eigenstate, another perhaps more interesting one would be not the neutrino itself, but a heayy neutral object like the WIMP we are looking for in our dark matter searches $\mathrm{l}$. If the objects do indeed exist they should be stable and if not overly massive, could be emitted in high energy bursts.

However, there is still the possibility of obtaining information even if the bursts don't separate into clearly distinguishable pulses corresponding to the different masses. There is still the original Zatsepin effect of the spreading of the pulse, here represented by Eq [5], and involving the same cosmological information as the separation effect Eq [4]. Note our distinction of the "separation" and "spreading" effects is for the purposes of a qualitative description and the two may well overlap, necessitating a detailed analysis including a modeling of the pulse shapes. Comparing the kinematic factors in Eq [4] and $\mathrm{Eq}$ [5] the condition that the mass separation $\Delta t$ be distinctly greater than that of the pulse spreading is $\frac{\Delta m^{2}}{2 m^{2}}>>\frac{\Delta p}{p}$, where $\Delta p$ is the energy spread of the burst. 


\section{Getting Into the Big Bang}

Observational methods using the photon, be it the optical photon of classical astronomy, the microwave photon of the background radiation or those of radio astronomy, will never allow us to look further back than "recombination", some 100,000 years after the Big Bang. One of the most intriguing potentialities of these neutrino or neutrino - like based observations is that these particles will come to us directly from a very early epoch. The "look-back time" is much deeper. Decoupling for the neutrino is in the first few minutes, so with neutrinos we can, in principle, go back to the first minutes and study the space-time geometry at that time.

Facing this exciting possibility of looking deep into the Big Bang are a few requirements and difficulties which are not exactly trivial. Again, some sort of phenomenon must exist around the epoch in question in which powerful high energy bursts of neutrinos are emitted. Perhaps collapse of overdense regions to black holes or annihilation of topological defects are a possibility. Then after being strongly redshifted and diluted by the expansion of the universe, the particles must be detected at the earth. Now we no longer have $z \leq \mathcal{O}(1)$ but rather, if we go all the way back to neutrino decoupling $z \sim(1 \mathrm{MeV} / 0.1 \mathrm{meV}) \sim$ $10^{10}$, so that these redshifts and dilutions will be very great. Furthermore, since the time scale of the event is stretched by $z$, a millisecond event will last a year upon reaching us. On the other hand this suggests something interesting: our grandchildren when operating very big neutrino arrays and observing long-time secular variations in the counting rate, should consider if they are perhaps seeing a burst out of the early universe and not an instability of their apparatus.

Alas, in view of the detection difficulties, this way of doing cosmology will have to probably remain fantasy for quite a while, if not forever. On the other hand, it is hard to think of any other way of directly looking at the pre-recombination epoch.

In a final mad fantasy we can of course push things even further back. Imagine that there is the WIMP or some other neutral, very weakly interacting particle, then we go even further back into the "Bang". Things like the formation of "Baby Universes", or other Colossal Happenings, presumably involving some activity and excitations of the particle fields on a microscopic timescale, become directly "visible" via their bursts, and allow us to study the space-time structure of the epoch. While this is indeed very far-fetched, it is amusing that at least at the level of fantasy, there is potentially an observational correspondence to such happenings. 


\section{References}

1. G. T. Zatsepin, ZhTEF Pis. Red. 8, 333 (1968), (JETP Lett. 8 205).

2. S. Pakvasa and K. Tennakone, Phys. Rev. Lett. 28, 1415 (1972); S. Pakvasa, DUMAND Symposium 1980; N. Cabibbo, Accademia Lincei, Meeting on Astrophysics and Elementary Particles, (1980); Tsvi Piran, Phys. Lett. B 102, 299 (1981); A.K. Drukier and L. Stodolsky,Phys. Rev. D 30, 2295 (1984). P. Reinartz and L. Stodolsky, Z.f.Phys. C27, 507 (1985).

3. For the discussion with respect to SN1987a see section 11.3.4 of G.G. Raffelt, Stars as Laboratories for Fundamental Physics, Univ. Chicago Press, (1996).

4. L. Stodolsky, Phys. Lett B 201, 353 (1988); M. J. Longo, Phys. Rev.D 36, 3276 (1987) and Phys. Rev. Lett. 60, 173 (1988); L. Krauss and S. Tremaine, Phys. Rev. Lett. 60, 176 (1988).

5. Y. Fukuda et al., (Super-Kamiokanda Collaboration), Phys. Rev. Lett. 81, 1562 (1998).

6. L. Stodolsky, Phys. Lett. B 473, 61 (2000).

7. S. Weinberg, Gravitation and Cosmology, chapt.14, John Wiley, New York, (1972).

8. S. P. Ahlen, F. T. Avignone lll, et al. Phys. Lett. B 195, 603 (1987), were the first to present laboratory limits on dark matter; for recent work see for example the CRESST report MPI-PTh/2000-04, or section Vl of Proceedings, LTD-8, P. De Korte and T.Peacock editors, North-Holland (2000). 